\title{
Protease-activated Receptor-2 Regulates Glial Scar Formation via JNK Signaling
}

\author{
Tian-Zun LI ${ }^{1}$, Qiang LIU ${ }^{2}$, Yong-Zhi XIA ${ }^{2}$, Rami DARWAZEH ${ }^{2}$, Yi YAN ${ }^{2}$ \\ ${ }^{1}$ Department of Neurosurgery, Daping Hospital and Institute Research of Surgery, Army Medical \\ University, Chongqing, China, ${ }^{2}$ Department of Neurosurgery, The First Affiliated Hospital of \\ Chongqing Medical University, Chongqing, China
}

Received March 16, 2018

Accepted October 22, 2018

Epub Ahead of Print January 10, 2019

\section{Summary}

The study aimed to determine the effects of protease-activated receptor-2 (PAR-2) on glial scar formation after spinal cord injury (SCI) in Sprague-Dawley (SD) rats and the underlying mechanisms. Rivlin and Tator's acute extradural clip compression injury (CCI) model of severe SCI was established in this study. Animals were divided into four groups: 1) sham group (laminectomy only); 2) model group, treated with normal saline; 3) PAR-2 inhibitor group; 4) PAR-2 activator group. Enhanced GFAP and vimentin expression were the markers of glial scar formation. To determine whether JNK was involved in the effects of PAR-2 on GFAP and vimentin expression, we administered anisomycin (a JNK activator) in the presence of PAR-2 inhibitor and SP600125 (a JNK inhibitor) in the presence of PAR-2 activator. At 1, 7, 14 and 28 day after SCI, Basso, Beattie, and Bresnahan (BBB) locomotor score test was used to assess the locomotor functional recovery; immunofluorescence and western blot analysis were used to assess the expression level of GFAP, vimentin and $p$-JNK. Double immunofluorescence staining with GFAP and tubulin $\beta$ was used to assess the glial scar formation and the remaining neurons. Results suggested that PAR-2 is involved in glial scar formation and reduces neurons residues which can cause a further worsening in the functional outcomes after SCI via JNK signaling. Therefore, it may be effective to target PAR-2 in the treatment of SCI.

\section{Key words}

Protease-activated receptor-2 • Spinal cord injury • Jun $\mathrm{N}$-terminal kinase

\section{Corresponding author}

Yi Yan. Department of Neurosurgery, The First Affiliated Hospital of Chongqing Medical University, No.1 Youyi Road, Yuzhong District, Chongqing 400016, China. E-mail: lihongphd@yeah.net

\section{Introduction}

Spinal cord injury (SCI) is a common disabling injury causing a huge psychological and social economic burden. Worldwide, an estimated 2.5 million people live with SCI, and more than 130,000 new injuries are reported each year (Wang et al. 2012). One of the main reasons leading to high morbidity and disablement after SCI is the formation of glial scar which presents a major obstacle for axonal regeneration (Wang et al. 2012, Qu et al. 2012, Hu et al. 2010). Suppression of glial scar formation is considered the target in the treatment of SCI (Hu et al. 2010). Therefore, better and more in-depth studies of the molecular mechanism for the regulation of glial scar formation should be made.

Protease-activated receptors (PARs) are a family of four $G$ protein-coupled receptors which include PAR-1, PAR-2, PAR-3 and PAR-4 (Bushell 2007, Luo et al. 2007, Park et al. 2009). PAR-2, which is widely distributed in the central nervous system (CNS), can be found in microglia (Noorbakhsh et al. 2006), astrocytes (Park et al. 2009) and neurons (Lohman et al. 2008). Also, it can be activated through proteolytic cleavage of its $\mathrm{N}$ termini by trypsin and mast cell tryptase (Park et al. 2009). Reactive astrogliosis is the major character of glial scar (Zhang et al. 2010). Meanwhile, studies suggest that PAR-2 may play an important role in reactive astrogliosis characterized by substantial astrocyte proliferation and enhanced GFAP and vimentin expression (Park et al. 2006, McCoy et al. 2010). On the basis of these findings, as well as the biological activities of PAR-2 in the 
proliferation of various other cell types (endometrial stromal cell, cardiac fibroblast, prostate cancer cell, human mesangial cell etc.) (Osuga et al. 2012, Ide et al. 2007, Mize et al. 2008), we tried to study the effects of PAR-2 on GFAP and vimentin expression, glial scar formation, neurons residues and behavioral recovery in the present study. And as far as we know, similar studies have not been done till now.

Mitogen-activated protein kinases (MAPKs) include extracellular signal-regulated kinase (ERK), Jun N-terminal kinase (JNK) and p38 MAPK (Ralay et al.2010). Now due to the key role of MAPK in cell proliferation (Irving et al. 2010, Guo et al. 2012) and the evidence that activation of PAR-2 can induce the phosphorylation of MAPK especially of JNK (Park et al. 2009, Park et al. 2010), we attempted to determine its role in the regulating effects of PAR-2 on the expression of GFAP, vimentin and functional outcomes.

\section{Methods}

\section{Ethics statement}

Sprague-Dawley (SD) female rats used in these experiments were housed in the laboratory animal center of Chongqing Medical University and all animal experiments were approved by the Institutional Animal Care and Use Committees of the First Affiliated Hospital of Chongqing Medical University (CMU-1-2633). All efforts were made to minimize the number of animals and their sufferings.

\section{Materials}

SD female rats weighing 210-230 $\mathrm{g}$ were purchased from the Laboratory Animal Center of Chongqing Medical University, Yasargil aneurysm clip was obtained from Rebstock (Tuttlingen, BW, Germany); mouse anti-GFAP antibody was obtained from BD Biosciences (catalog, 556327, San Diego, CA, USA); rabbit anti-tubulin $\beta$ polyclonal antibody was purchased from ImmunoWay Biotechnology (catalog,YM3247, Newark, DE, USA); Anti-PAR-2 antibody (catalog, ab184673), PAR-2 agonist (SLIGRL-NH2, SL), antivimentin antibody(catalog, ab8978) and goat polyclonal secondary antibody to mouse IgG1-heavy chain (catalog, ab97240) were obtained from Abcam Inc (Cambridge, MA, USA); PAR-2 inhibitor, FSLLRY-NH2 (FS) was purchased from PEPTIDES INTERNATIONAL Inc (Louisville, KY, USA); JNK agonist (anisomycin, AN) was obtained from Sangon Biotech (Shanghai) Co., Ltd.
(Shanghai, SH, China); JNK inhibitor (SP600125, SP), GAPDH antibody was obtained from Beyotime Institute of Biotechnology (catalog, AG019, Haimen, JS, China); phospho-SAPK/JNK (Thr183/Tyr185) antibody (catalog, 9251S) and SAPK/JNK antibody (catalog, 9252) were obtained from Cell Signaling Technology (Beverly, MA, USA); flur ${ }^{\circledR}$ 488-conjugated affiniPure goat anti-mouse IgG (H+L) antibody (catalog, 33206ES60) and peroxidase-conjugated affiniPure goat anti-rabbit $\operatorname{lgG}$ $(\mathrm{H}+\mathrm{L})$ antibody were purchased from ZSGB-BIO (catalog, 111-035-003); Beijing, BJ, China. The rest of the reagents were obtained from Beyotime Institute of Biotechnology (Haimen, JS, China) and the Neuroscience research center of Chongqing Medical University.

\section{Model of severe SCI}

Rivlin and Tator's acute extradural clip compression injury (CCI) model was used for producing severe SCI (Usul et al. 2006). SD rats were anesthetized with $10 \%$ chloral hydrate $(3.5 \mathrm{ml} / \mathrm{kg})$ and breathing spontaneously without tracheal intubation. After laminectomy of T11-T12, CCI model was performed with a $0.88 \mathrm{~N}$ closing force aneurysm clip for $1 \mathrm{~min}$. All injured animals were paralyzed immediately after SCI. Following SCI, a catheter $(6-8 \mathrm{~cm}$ with a diameter $1 \mathrm{~mm})$ was fixed in intradural of the T11-T12, with one end was externalized behind the neck for access during dosing (Xia et al. 2008). All surgical processes had been done aseptically and the animals were placed on a heating pad to maintain their body temperature during the operation. Under inhalating anesthesia (2-3 $\mathrm{ml}$ of liquid sevoflurane), the animal had been slowly injected with the treatment agents every other day for 2 weeks via the catheter using a mechanical syringe driver (Germany HENKE-SASS continuous syringe $1 \mathrm{ml}$ ) over $10 \mathrm{~min}$. The wounded rats were received bladder compression three times a day. And all the rats were sacrificed at 1, 7, 14 and $28 \mathrm{~d}$ after SCI respectively. The SCI for all animal models had been performed by the same professional person in the same experimental conditions to reduce the possibility of experimental errors. Also, the same aneurysm clamp was used for all animal models to ensure the consistency of SCI.

\section{Experimental grouping and intrathecal interventions}

FS is the specific PAR-2 inhibitor; SL is the specific PAR-2 activator; AN is the specific JNK activator; SP is the specific JNK inhibitor. Following the laminectomy, rats were randomly divided into four 
groups and each group had 24 rats with 6 rats at each time point: 1) sham group: only laminectomy was performed; 2) model group: following laminectomy and SCI, $10 \mu \mathrm{l}$ normal saline (NS) was administered each time; 3) SL group: following laminectomy and SCI, $10 \mu \mathrm{l}$ SL $(50 \mu \mathrm{M}$, diluted in NS) was administered each time; 4) FS group: following laminectomy and SCI, $10 \mu \mathrm{FS}$ (50 $\mu \mathrm{M}$, diluted in NS) was administered each time. To explore the role of JNK in the effects of PAR-2 on the function outcomes and the expression of GFAP and Vimentin, following the laminectomy rats were randomly divided into four groups and each group had 24 rats with 6 rats at each time point: 1) sham group: only laminectomy was performed; 2) model group: following laminectomy and SCI, $1 \%$ DMSO $(10 \mu \mathrm{l})$ was administered each time; 3) $\mathrm{FS}+\mathrm{AN}$ (diluted in $1 \%$ DMSO) group: following laminectomy and SCI, $10 \mu \mathrm{l}$ mixture of FS $(50 \mu \mathrm{M})$ and $\mathrm{AN}(20 \mu \mathrm{M})$ was administered each time; 4) SL+SP (diluted in $1 \%$ DMSO): following laminectomy and SCI , $10 \mu \mathrm{l}$ mixture of SL $(50 \mu \mathrm{M})$ and SP $(30 \mu \mathrm{M})$ was administered each time. The concentrations of the above regents were selected depending on the previous study and experiments in advance.

\section{Immunofluorescence}

Animals were perfused with NS after being anesthetized with a lethal dose of chloral hydrate, followed by perfusion fixation with $4 \%$ paraformaldehyde diluted in $0.1 \mathrm{M}$ PBS. $1 \mathrm{~cm}$ of spinal cord centered at the lesion site (or equivalent location in sham operation animals) was immediately isolated and then fixed with $4 \%$ paraformaldehyde for $12 \mathrm{~h}$. After gradient alcohol dehydration, the spinal cords were embedded in paraffin and sectioned longitudinally at $5 \mu \mathrm{m}$ thickness. After deparaffinization and hydration, the tissue slides were repaired with citrate buffer at $96{ }^{\circ} \mathrm{C}$ for $30 \mathrm{~min}$ and then cooled to room temperature naturally. After blocking with endogenous peroxidase blocking buffer for $10 \mathrm{~min}$ at room temperature, slides were rinsed three times in PBS, then blocked with $10 \%$ normal goat serum at $37{ }^{\circ} \mathrm{C}$ for $50 \mathrm{~min}$. To detect the extent of astrogliosis, slides were incubated with a mouse monoclonal antibody for GFAP (1:50) for $24 \mathrm{~h}$. To assess the glial scar formation and axonal regeneration, the rabbit polyclonal antibody for tubulin $\beta$ (1:400) was applied in combination with GFAP primary antibody (1:50) for $24 \mathrm{~h}$. After being washed three times with PBS, slides were incubated with goat anti-mouse IgG fluorescence secondary antibody (1:200) and/or goat anti- rabbit IgG fluorescence secondary antibody (1:200) at $37^{\circ} \mathrm{C}$ for $1 \mathrm{~h}$. The results were then analyzed using a fluorescence microscope (Olympus Microsystems) and confocal microscopy (LEICA TCS SP2). The extent of astrogliosis was measured as the GFAP positive staining area ratio (GFAP positive staining area/total area of tissue) (Wang et al. 2012, Noorbakhsh et al. 2006). Meanwhile, the glial scar thickness means the average glial scar thickness of the glial scar on both sides and the midline of spinal cord, and was measured as the average distance between the edge of the cavity and the edge of the rostral/caudal and lateral glial scar. The more glial scar was formed, the thicker the glial scar would be. All measurements described above were measured with Image-Pro plus 6.0 software.

\section{Western blot}

Animals were anesthetized with a lethal dose of chloral hydrate. $1 \mathrm{~cm}$ of spinal cord centered at the lesion site (or equivalent location in sham operation animals) was immediately dissected and then homogenized in the ice-cold mixture (1g tissue/7.5ml mixture) of PMSF and RIPA lysis (50 mM Tris, pH 7.4, $1 \%$ Triton X-100, $1 \%$ sodium deoxycholate, $0.1 \% \mathrm{SDS}, 30 \mathrm{mM}$ sodium fluoride, $5 \mathrm{mM}$ EDTA, $1 \mathrm{mM}$ sodium orthovanadate, $10 \mathrm{M}$ leupeptin). The supernatant was collected after a centrifugation at $10,000 \mathrm{~g}$ for $15 \mathrm{~min}$ at $4{ }^{\circ} \mathrm{C}$. Protein concentrations were determined by BCA assay. Approximately $50 \mu \mathrm{g}$ protein sample run on a $10 \%$ SDSPAGE electrophoresis gel and transferred to a PVDF membrane. The membranes were blocked with specialized western blocking buffer and were incubated with the following primary antibodies: anti-GFAP (1:500), anti-vimentin (1:1500), anti- p-JNK (1:1000), anti-JNK (1:1000), or anti-GAPDH $(1: 1000)$ at $4{ }^{\circ} \mathrm{C}$ overnight. After being washed three times in PBST, the membranes were incubated with goat anti-mouse IgG secondary antibody $(1: 1000)$ or goat anti-rabbit $\operatorname{lgG}$ secondary antibody $(1: 1000)$ at $37^{\circ} \mathrm{C}$ for $2 \mathrm{~h}$. The membranes were then colored with $\mathrm{DAB}$ for 1-2 min at room temperature.

\section{Behavioral analysis}

In our study, before the rats been sacrificed, Basso, Beattie, Bresnahan locomotor rating scale (BBB) was used to assess functional outcomes of rat's hind limbs at the 1, 7, 14 and 28 day after SCI (Basso 2004). In brief, animals were placed into an open-field environment consisting of a Plexiglass arena and scored 
by two observers blinded to the treatment for a period of $4 \mathrm{~min}$. To ensure no deficits in rat's hind limbs function, animals were also assessed before SCI in an open-field testing environment.

\section{Statistical analysis}

Statistical analysis was performed using SPSS 19 Statistics software and results presented as means \pm SEM. The evaluation of GFAP, Vimentin expression, p-JNK, and glial scar thickness were carried out by one-way ANOVA within multiple groups and by least significant difference (LSD) or Tamhane's T2 respectively depending on the homogeneity of variance between two groups. BBB score evaluation was completed by repeated-measures ANOVA within multiple groups and Bonferroni post-hoc analysis between two groups. Differences with a P value less than 0.05 were considered significant.

\section{Results}

$P A R-2$ regulated the reactive astrogliosis in the lesion site of spinal cord after SCI

As shown in the Fig. 1, PAR2 expression peaked on the 1st day after SCI then gradually decreased, which was still significantly higher than that in normal animals on $28^{\text {th }}$ day. Next, the expression level of GFAP after SCI with various interventions was assayed by western blot. The expression level of GFAP after SCI with JNK agonist AN was gradually increased. However, with JNK inhibitor SP was decreased gradually (Fig. 2).

GFAP immunofluorescence staining was performed to determine whether PAR-2 can regulate the reactive astrogliosis at the lesion site of the rat's spinal cord. As shown in Fig. 3, at the 7, 14 and 28 day after $\mathrm{SCI}$, the GFAP positive staining area ratio in model group was significantly larger than that in FS group but less than that in SL group.

\section{PAR-2 regulated the expression of GFAP and Vimentin in injured rat's spinal cord}

To determine whether PAR-2 can regulate the expression of GFAP and vimentin in injured rat's spinal cords, the lesion site of the rat's spinal cords were exposed to FS $(50 \mu \mathrm{M})$ or SL $(50 \mu \mathrm{M})$ for 14 days after SCI. Additionally, at the $1,7,14$ and 28 day after SCI, the expression level of GFAP and vimentin was measured by western blot. As shown in Fig. 4, at the 7, 14 and 28 day after SCI, the expression level of GFAP and vimentin in model group is significantly higher than that in FS group but significantly lower than that in SL group (Fig. 4).

Formation of glial scar and neurons residues in injured rat's spinal cords

Double immunofluorescent staining with GFAP and tubulin $\beta$ was performed to determine the effects of PAR-2 on glial scar formation and neurons residues. As shown in Fig. 5, at the 28 day after SCI, the GFAPpositive astrocytes in model group are significantly more than those in FS group but less than those in SL group. In addition, in sham group, numerous tubulin $\beta$-positive neurons and axons were in regular organization. However, in model group, the tubulin $\beta$-positive neurons and axons around the cavity wall were less, and the neurons body did not appear hypertrophic and some axons could be seen penetrating the glial scar to the cavity wall. In FS group, the tubulin $\beta$-positive axons and neurons around the cavity wall were abundant, the neurons body appeared hypertrophic significantly and most axons could be seen penetrating the glial scar even to the cavity wall. In SL group, no specific tubulin $\beta$-positive neurons and axons were appeared at the same location as those in model and FS groups (Fig. 5). Meanwhile, the thickness of glial scar in model group were significantly thicker than that in FS group but thinner than that in SL group. As shown in Table 1, the glial scar thickness was $551.64 \pm 116.64 \mu \mathrm{m}$ in model group, $371.24 \pm 79.22 \mu \mathrm{m}$ in FS group and 931.32 \pm $178.29 \mu \mathrm{m}$ in SL group.

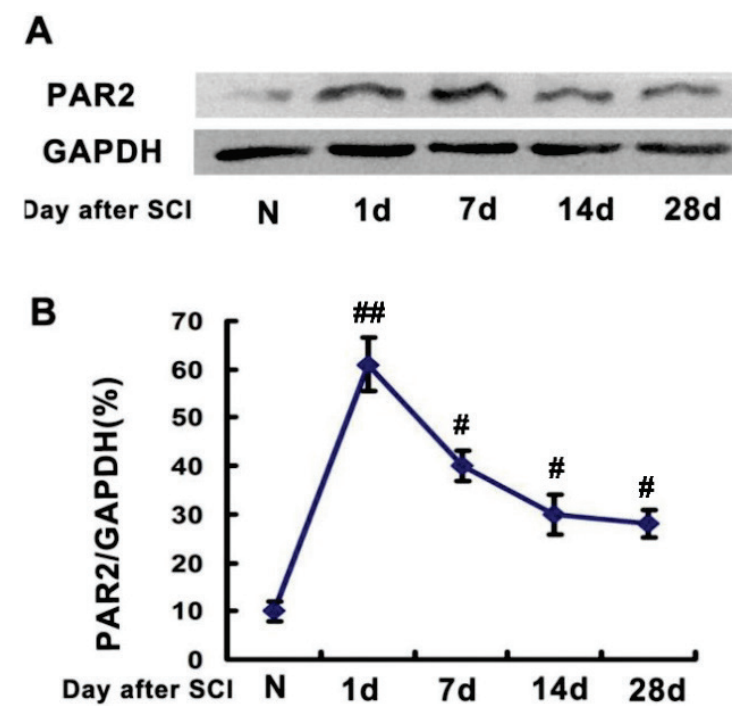

Fig. 1. Expression level of PAR2 after SCI without any intervention $(\mathbf{n = 6})$. PAR2 expression peaked on the $1^{\text {st }}$ day and then gradually decreased after SCI. N, normal spinal cord. ${ }^{\# \#} \mathrm{p}<0.01$ and ${ }^{\#} \mathrm{p}<0.05$ vs. $\mathrm{N}$. 
A

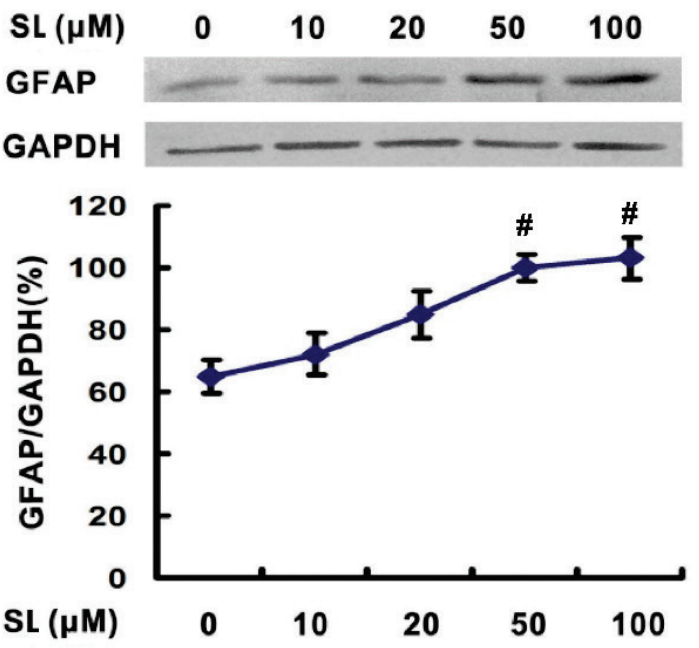

C
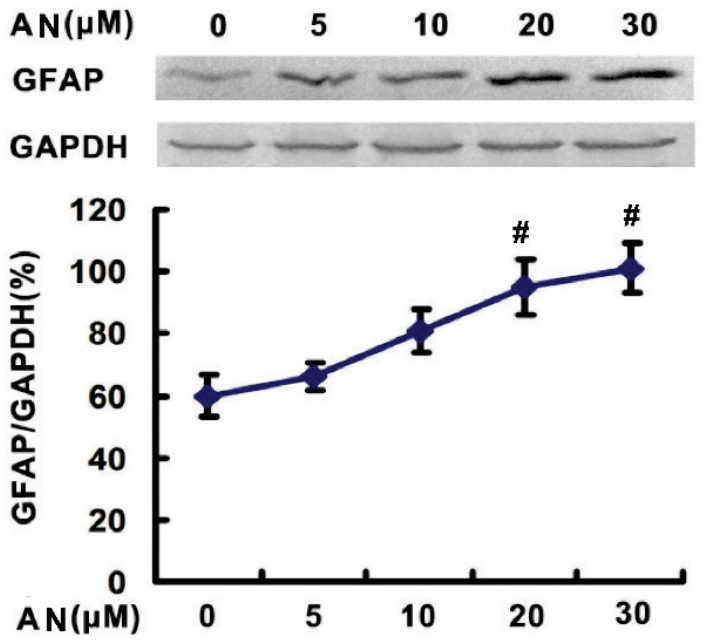

B

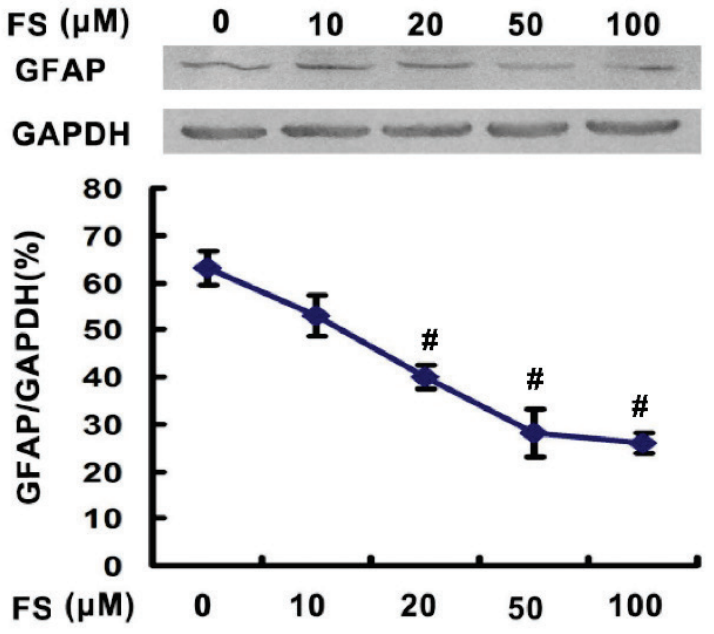

D

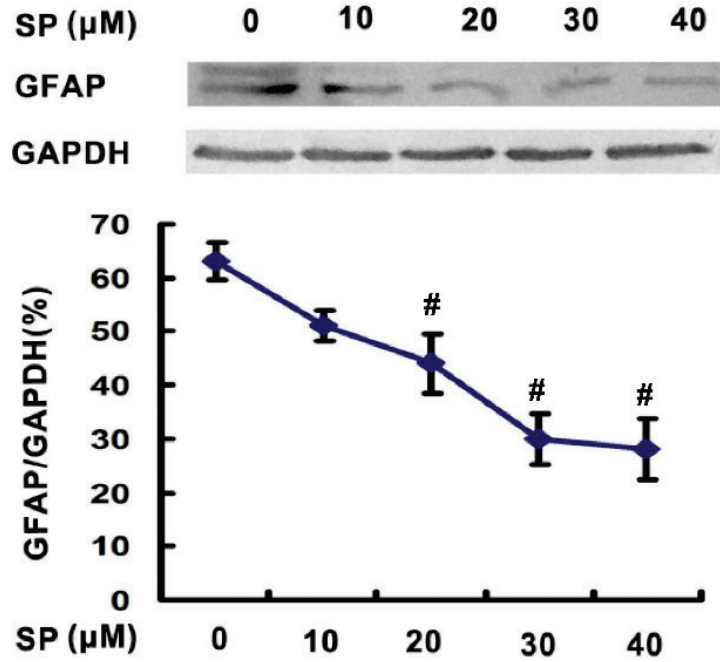

Fig. 2. The expression level of GFAP after SCI with various interventions $(\mathbf{n}=\mathbf{6})$. (A) the expression level of GFAP after SCI with PAR2 agonist SL; (B) the expression level of GFAP after SCI with PAR2 inhibitor FS; (C) the expression level of GFAP after SCI with JNK agonist AN; (D) the expression level of GFAP after SCI with JNK inhibitor SP. ${ }^{\#} p<0.05$ vs. 0.

\section{$B B B$ score of rat's hind limbs}

BBB score was used to assess functional recovery of rats' hind limbs at the $1,7,14$ and 28 days after SCI. As shown in Table 2, the BBB scores of the rat's hind limbs before SCI were identical $(21.00 \pm 0.00)$. And at the 7, 14 and $28 \mathrm{~d}$ after SCI, the BBB score in model group was significantly higher than that in FS group but significantly lower than that in SL group.

\section{JNK signaling was involved in PAR-2 activation}

As shown in Fig. 6 when compared with model group, treatment with FS significantly prevented the increase of $\mathrm{p}-\mathrm{JNK}$ expression, while treatment with SL significantly boosted the increase of $\mathrm{p}-\mathrm{JNK}$ expression at the 1,7 and 14 day after SCI.

Table 1. Quantitative measurement of the glial scar thickness $(n=6)$.

\begin{tabular}{ll}
\hline Groups & Glial scar thickness $(\boldsymbol{\mu m})$ \\
\hline Model group & $551.64 \pm 116.64$ \\
FS group & $371.24 \pm 79.22^{\#}$ \\
SL group & $931.32 \pm 178.29^{\# \#}$
\end{tabular}

The quantitative measurement of the glial scar thickness. The results shown are the mean \pm SEM of three independent experiments. ${ }^{\# \#} p<0.01$ and ${ }^{\#} p<0.05$ vs. model group. 
A

so
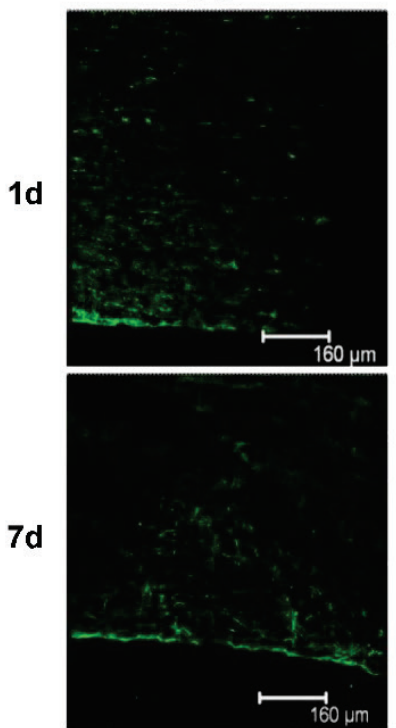

$160 \mathrm{~mm}$
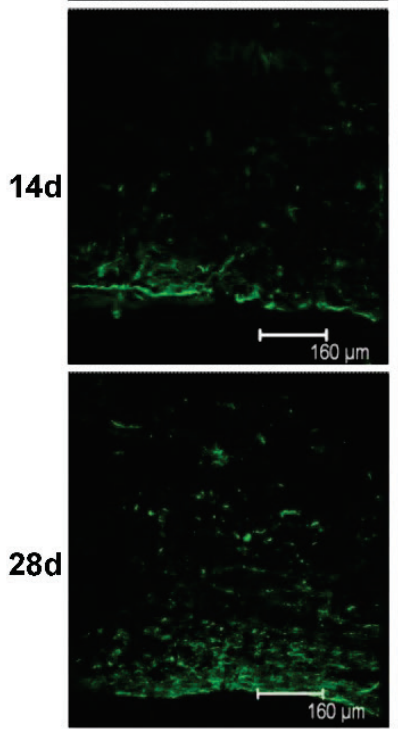

B
MO
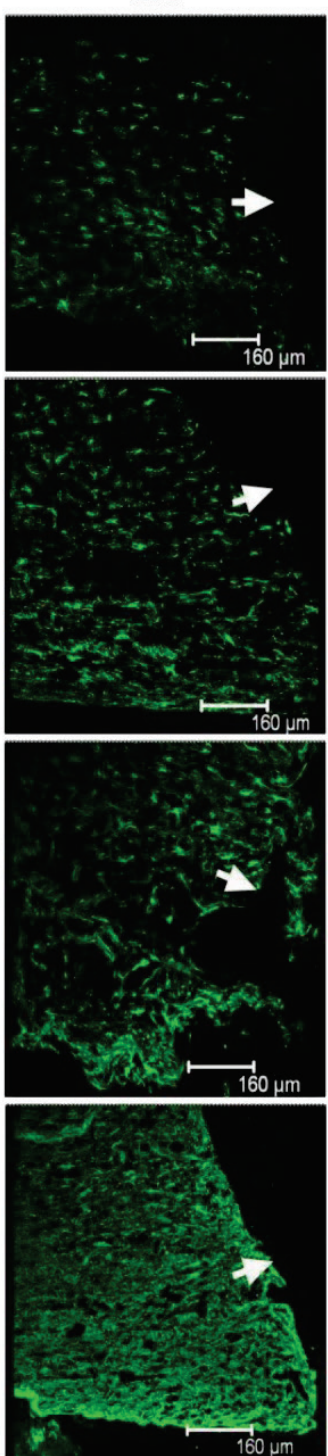

$\square$ SO $\square$ MO $\square$ FS $\square$ SL
FS
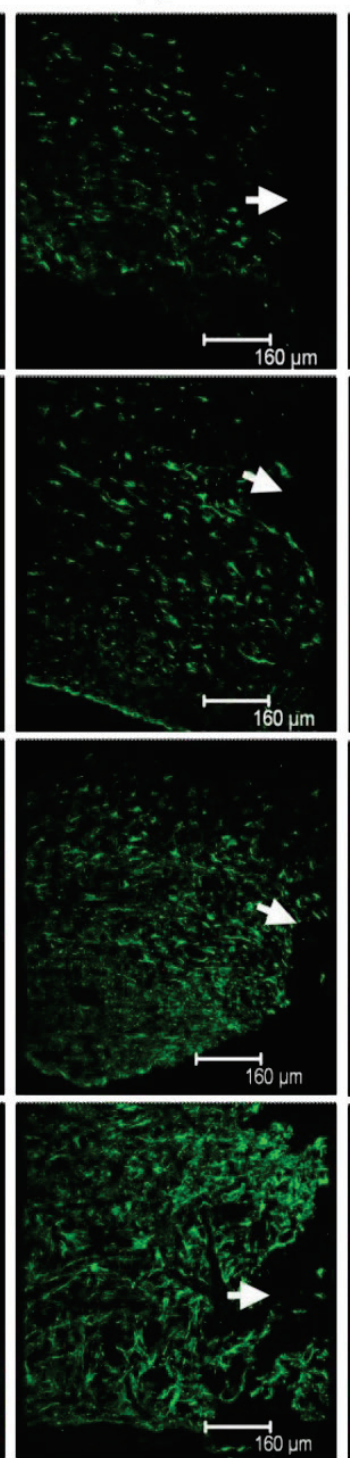

SL
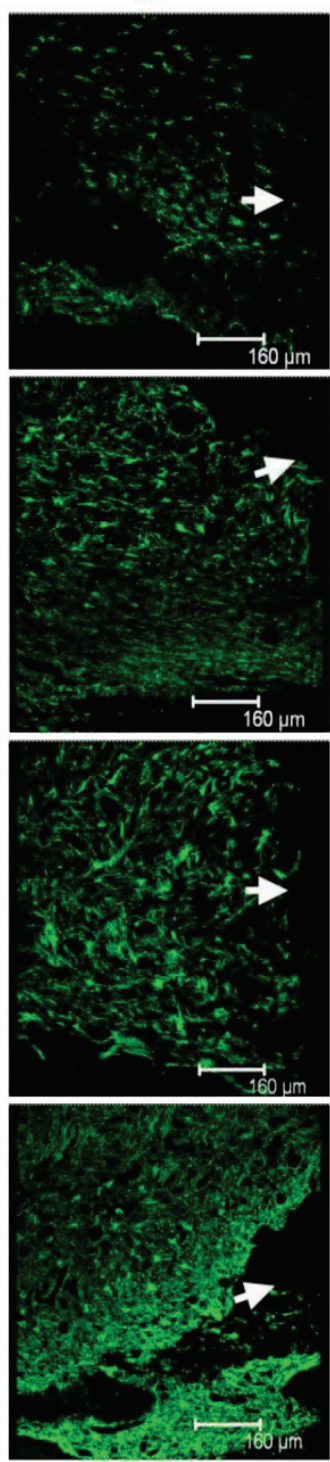

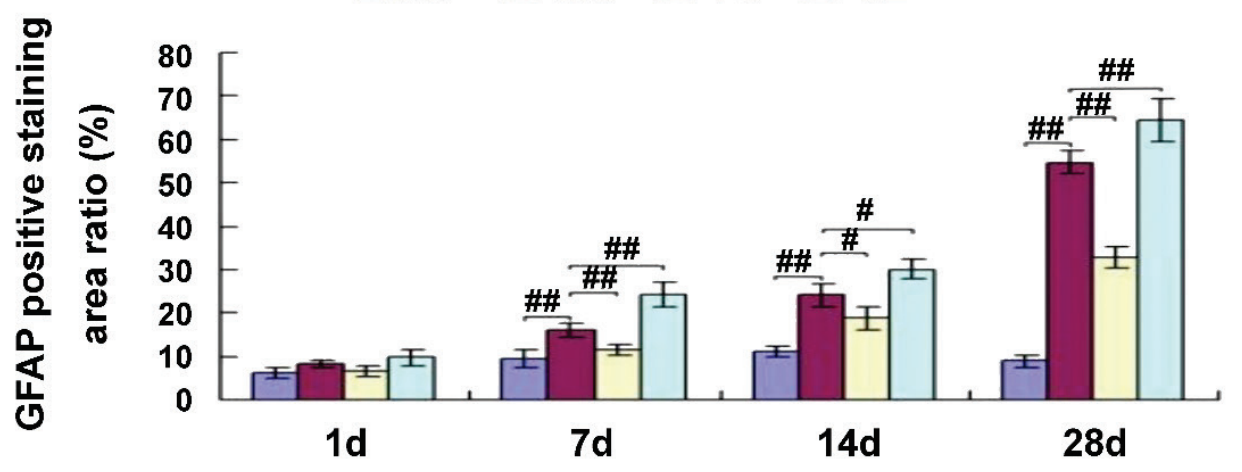

Fig. 3. PAR2 regulates GFAP expression $(\mathbf{n = 6})$. (A) Confocal images of injured spinal cords on the 1, 7, 14 and 28 day after SCI, which were treated with FS $(50 \mu \mathrm{M})$ or SL $(50 \mu \mathrm{M})$ for 14 days after SCI. (B) Quantification of GFAP positive staining area ratio in injured spinal cord exposed to FS or SL on the $1,7,14$ and 28 day after SCI. ${ }^{\#} p<0.01$ and ${ }^{\#} p<0.05$ vs. SO. SO, sham group; MO, model group. The injured location presented as white arrows. 
A

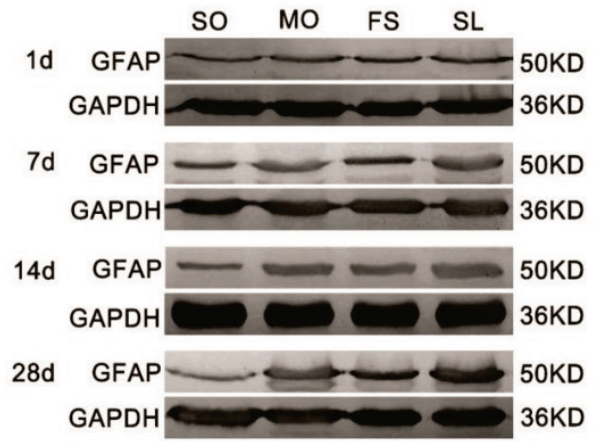

B

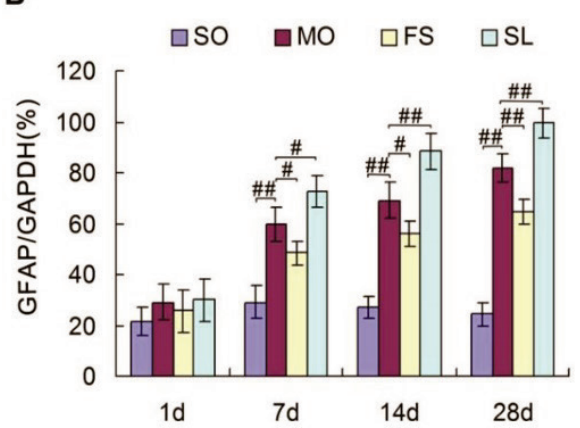

C

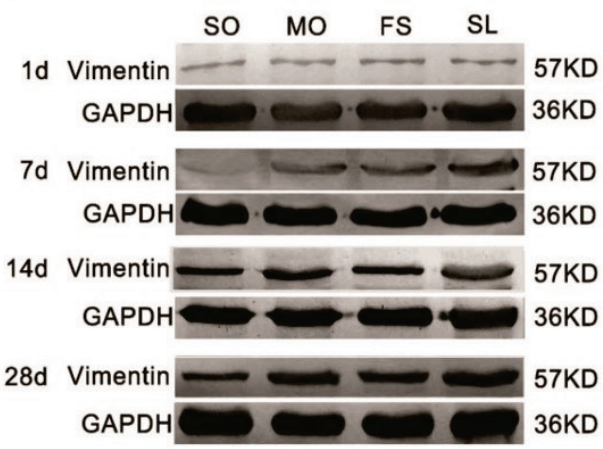

D

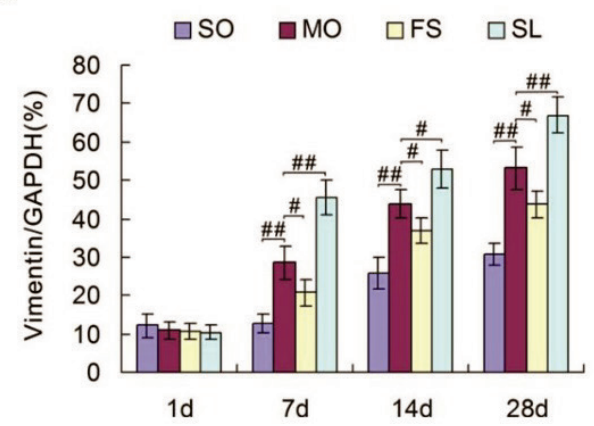

Fig. 4. PAR2 regulates GFAP and Vimentin expression $(\mathbf{n = 6}) .(A, C)$ The expression of GFAP and Vimentin in injured spinal cords at the $1,7,14$ and 28 day after SCI, which were treated with FS $(50 \mu \mathrm{M})$ or $\mathrm{SL}$ $(50 \mu \mathrm{M})$ for 14 days after SCI. (B, D) Quantification of GFAP and Vimentin expression in injured spinal cord exposed to FS or SL on the $1,7,14$ and 28 day after SCI. Data are the mean \pm SEM of three independent experiments. \#\# $\mathrm{p}<0.01$ and \# $\mathrm{p}<0.05$ vs. MO. SO, sham operation group; $\mathrm{MO}$, model group.

Fig. 5. Formation of glial scar and neurons residues in injured rat's spinal cord (GFAP [green] and tubulin $\beta$ [red]). Images of injured spinal cords on 28 day after SCI, which were treated with FS $(50 \mu \mathrm{M})$ or SL $50 \mu \mathrm{M}$ ) for $14 \mathrm{~d}$ after SCI. The tubulin $\beta$-positive neurons (blue arrows) and axons (white arrows) were shown in each group. The white dotted lines represent the boundary between glial scar and relative normal spinal cord. 
A

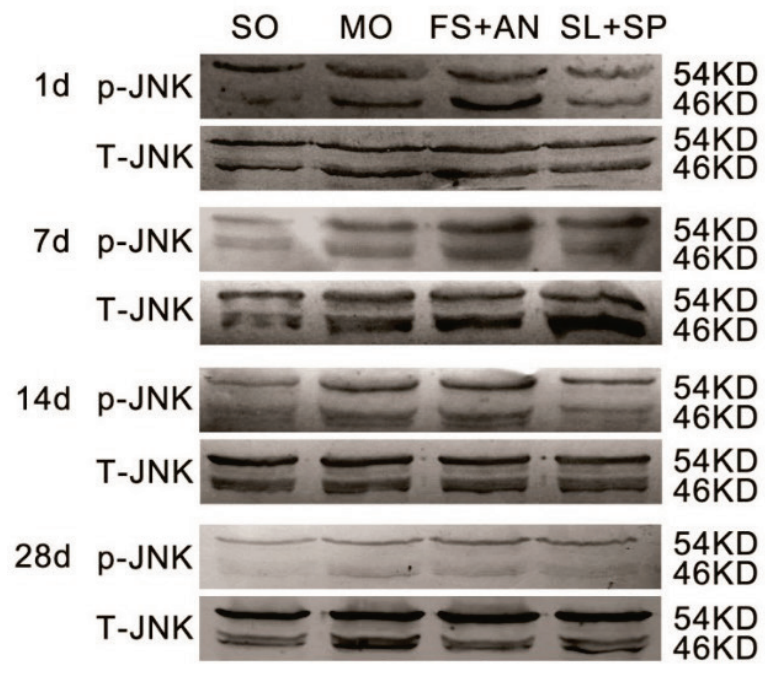

B

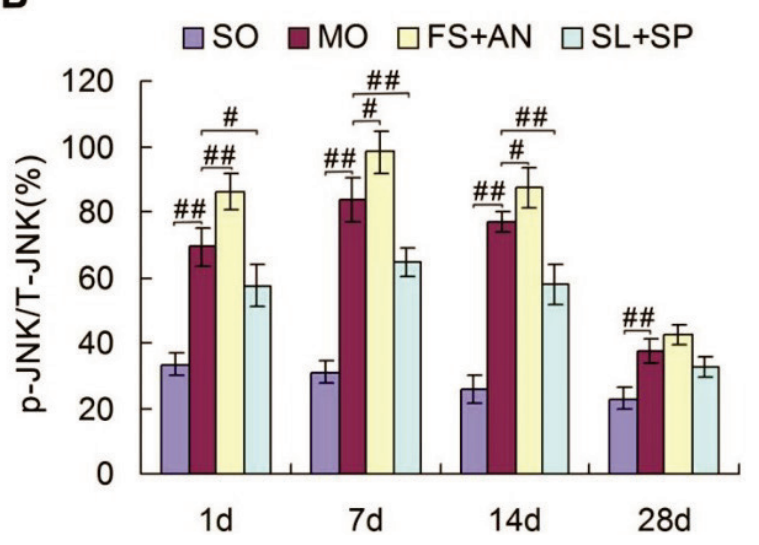

Fig. 6. PAR2 regulates $p-J N K$ expression without any intervention $(\mathbf{n}=\mathbf{6})$. (A) The expression of $\mathrm{p}$-JNK in injured spinal cords on the 1, 7, 14 and 28 day after SCI, which were treated with FS $(50 \mu \mathrm{M})$ or SL $(50 \mu \mathrm{M})$ for 14 days since SCI. (B) Quantification of p-JNK expression on injured spinal cord exposed to FS or SL on the $1,7,14$ and 28 day after SCI. \#\# $p<0.01$ and \# $p<0.05$ vs. model group. SO, sham operation group; MO, model group; T-JNK, total JNK.
A

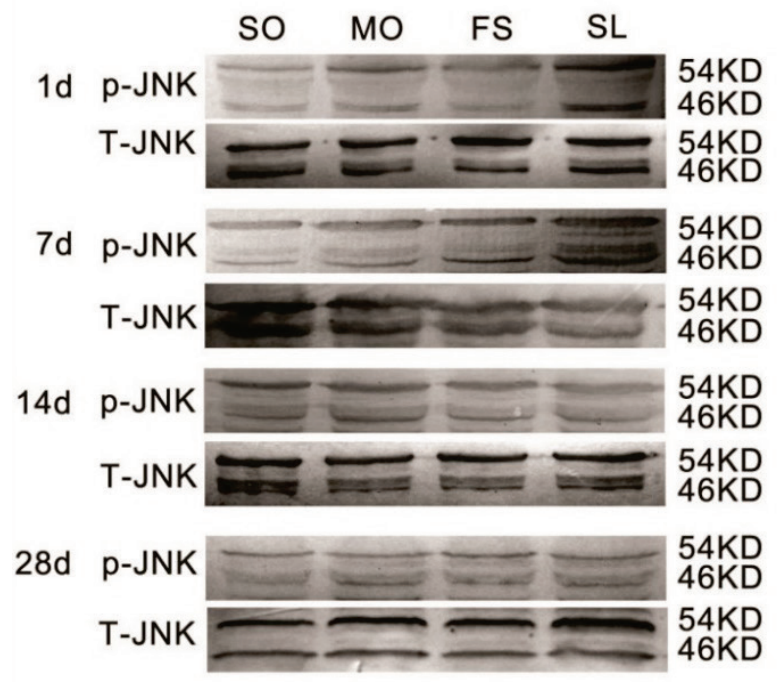

B

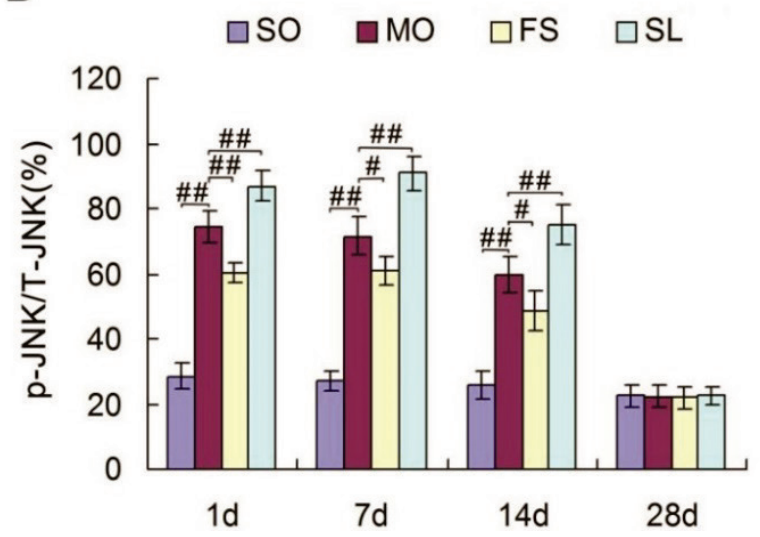

Fig. 7. JNK signaling was involved in PAR-2 activation in injured rat's spinal cord $(\mathbf{n}=\mathbf{6})$. $(\mathbf{A})$ The expression of $\mathrm{p}$-JNK in injured spinal cords on the $1,7,14$ and 28 day after SCI, which were treated with $\mathrm{FS}(50 \mu \mathrm{M})+\mathrm{AN}(20 \mu \mathrm{M})$ or SL $(50 \mu \mathrm{M})$ $+\mathrm{SP}(30 \mu \mathrm{M})$ for 14 days. (B) Quantification of p-JNK expression in injured spinal cord exposed to FS+AN or SL+SP on the 1,7 , 14 and 28 day after SCI. \#\# $\mathrm{p}<0.01$ and ${ }^{\#} \mathrm{p}<0.05$ vs. model group. SO, sham operation group; MO, model group; T-JNK, total JNK.

Table 2. BBB score of rats hind limbs $(n=6)$

\begin{tabular}{llllll}
\hline Groups & Before SCI & $\mathbf{1}$ day & $\mathbf{7}$ day & $\mathbf{1 4}$ day & $\mathbf{2 8 ~ d a y ~}$ \\
\hline Sham group & $21.00 \pm 0.00$ & $20.5 \pm 0.55^{\# \#}$ & $21.00 \pm 0.00^{\# \#}$ & $21.00 \pm 0.00^{\# \#}$ & $21.00 \pm 0.00^{\# \#}$ \\
Model group & $21.00 \pm 0.00$ & $0.67 \pm 0.52$ & $4.67 \pm 0.82$ & $6.33 \pm 0.82$ & $7.83 \pm 1.17$ \\
FS group & $21.00 \pm 0.00$ & $0.67 \pm 0.52$ & $6.17 \pm 0.75^{\#}$ & $8.0 \pm 0.89^{\#}$ & $9.83 \pm 0.75^{\# \#}$ \\
SL group & $21.00 \pm 0.00$ & $0.67 \pm 0.52$ & $2.67 \pm 0.82^{\#}$ & $4.67 \pm 0.82^{\#}$ & $6.33 \pm 1.03^{\# \#}$ \\
\hline
\end{tabular}

The BBB score of rat's hind limbs before and on the $1,7,14$ and 28 day after SCI. ${ }^{\# \#} p<0.01$ and ${ }^{\#} p<0.05$ vs. model group. 
Table 3. BBB score of rats hind limbs $(n=6)$

\begin{tabular}{llllll}
\hline & Before SCI & $\mathbf{1}$ day & $\mathbf{7}$ day & $\mathbf{1 4}$ day & $\mathbf{2 8}$ day \\
\hline Sham group & $21.00 \pm 0.00$ & $20.5 \pm 0.55^{\# \#}$ & $21.00 \pm 0.00^{\# \#}$ & $21.00 \pm 0.00^{\# \#}$ & $21.00 \pm 0.00^{\# \#}$ \\
Model group & $21.00 \pm 0.00$ & $0.67 \pm 0.52$ & $4.50 \pm 0.84$ & $6.50 \pm 0.55$ & $7.83 \pm 1.32$ \\
FS + AN group & $21.00 \pm 0.00$ & $0.67 \pm 0.52$ & $2.83 \pm 0.75^{\#}$ & $5.17 \pm 0.75^{\#}$ & $5.83 \pm 0.75^{\# \#}$ \\
SL + SP group & $21.00 \pm 0.00$ & $0.67 \pm 0.52$ & $6.17 \pm 0.75^{\#}$ & $8.5 \pm 1.05^{\#}$ & $9.83 \pm 0.41^{\# \#}$ \\
\hline
\end{tabular}

The BBB score of rat's hind limbs before and on the $1,7,14$ and 28 day after SCI. ${ }^{\# \#} p<0.01$ and ${ }^{\#} p<0.05$ vs. model group.

A

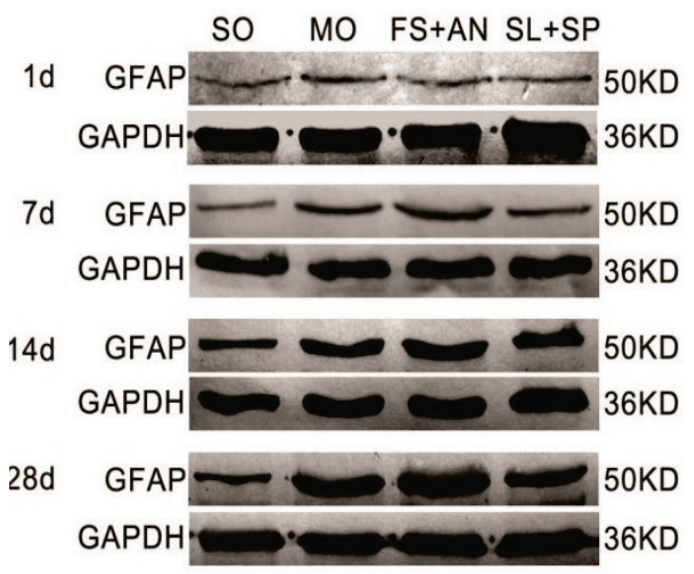

B

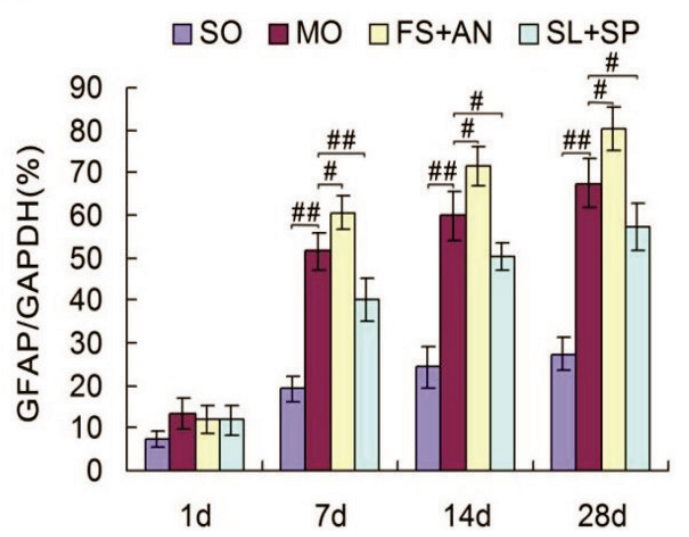

C

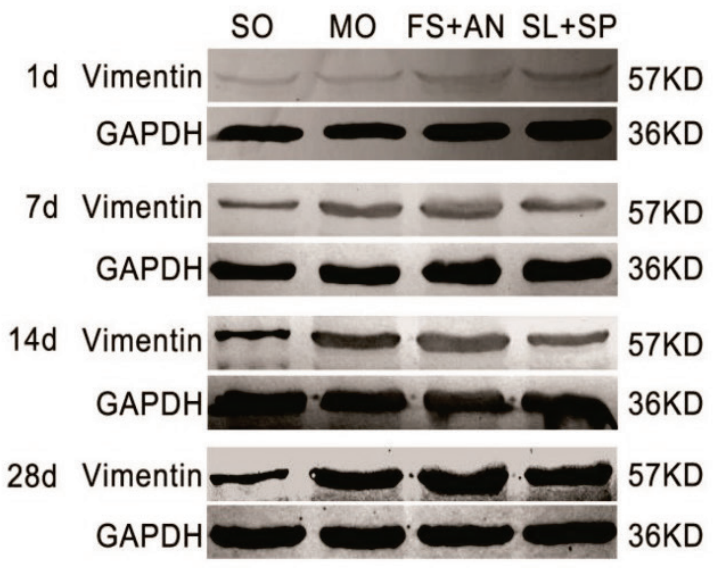

D

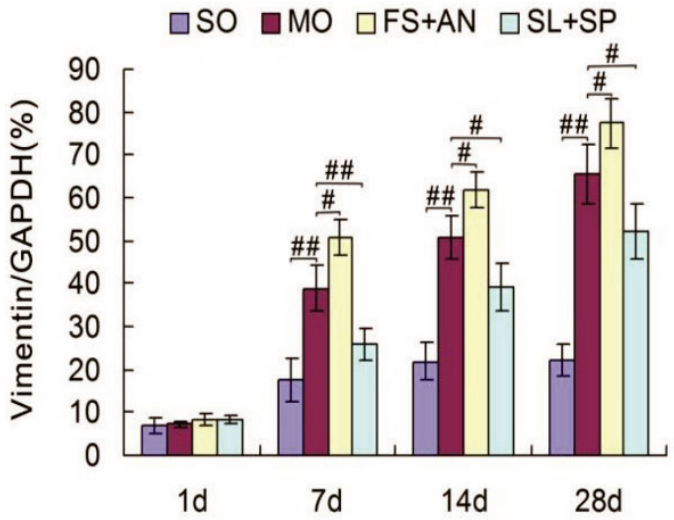

Fig. 8. Intervention of PAR-2 and JNK affects GFAP and Vimentin expression in injured rat's spinal cord $(n=6)$. (A, C) The expression of GFAP and Vimentin in injured spinal cords on the 1, 7, 14 and 28 day after SCI, which were treated with FS ( $50 \mu \mathrm{M})$ + AN $(20 \mu \mathrm{M})$ or SL $(50 \mu \mathrm{M})+\mathrm{SP}(30 \mu \mathrm{M})$ for $14 \mathrm{~d}$ after SCI. (B, D) Quantification of GFAP and Vimentin expression on injured spinal cord exposed to FS +AN or SL + SP on the $1,7,14$ and $28 d$ after SCI. ${ }^{\#} p<0.01$ and ${ }^{\#} p<0.05$ vs. model group. SO, sham operation group; MO, model group.

To determine whether JNK was involved in the regulating effects of PAR-2 on the expression of GFAP and vimentin, we administrated AN in the presence of FS or SP in the presence of SL for 14 days after SCI. At the 1, 7, 14 and 28 day after SCI, we measured the p-JNK expression in the injured spinal cords. As shown in Fig. 7, when compared with model group, treatment with
$\mathrm{SL}+\mathrm{SP}$ significantly prevented the increase of p-JNK expression, while treatment with $\mathrm{FS}+\mathrm{AN}$ significantly boosted the increase of $\mathrm{p}-\mathrm{JNK}$ expression at the 1,7 and 14 day after SCI.

Next, we measured the expression of GFAP and vimentin in the presence of co-administration of FS with AN or co-administration of SP with SL for $14 \mathrm{~d}$ since 
SCI. As shown in Fig. 8, treatment with FS+AN significantly boosted the increase of GFAP and Vimentin expression, while treatment with SL+SP significantly prevented the increase of GFAP and vimentin expression at the 7,14 and $28 \mathrm{~d}$ after SCI.

$B B B$ scores of rat's hind limbs after the Co-intervention of PAR-2 and JNK

To assess the functional recovery of rat's hind limbs in the presence of co-administration of FS with AN or co-administration of SP with SL for $14 \mathrm{~d}$ after SCI, we made a BBB score of rat's hind limbs at the 1, 7, 14 and $28 \mathrm{~d}$ after SCI. As shown in Table 3, the BBB score of rat's hind limbs before SCI is identical $(21.00 \pm$ 0.00 points). While at the 7,14 and $28 \mathrm{~d}$ after SCI the BBB score in model group was significantly higher than that in FS+AN group, but significantly lower than that in $\mathrm{SL}+\mathrm{SP}$ group.

\section{Discussion}

Glial scar formation, the major physical impediment for axonal regeneration, is characterized by reactive astrogliosis with enhanced GFAP and vimentin expression (Wang et al. 2012, Qu et al. 2012, Hu et al. 2010). Meanwhile, our previous study had proved the direct relation between glial scar formation and the expression of GFAP and vimentin (Liu et al. 2012). To prove the effects of PAR-2 on the expression of GFAP and vimentin, we found that the expression levels of the two proteins in model group were significantly higher than those in FS group but lower than those in SL group after SCI. So, we suggested that PAR-2 can regulate the expression of the two proteins after SCI. In addition, we found that inhibition of PAR-2 activity reduces glial scar formation, improves neurons residues and functional outcomes. Also promotion of PAR-2 activity boosts glial scar formation, reduces neurons residues and worsens functional outcomes. Thus, after a comprehensive analysis of all of the above results, we concluded that PAR-2 exerts the regulating effects on glial scar formation by regulating GFAP and vimentin expression.

Reactive astrogliosis as the major characteristic of glial scar formation is a complex phenomenon that includes a mixture of positive and negative responses for neuronal survival and regeneration (Sofroniew and Vinters 2010). In early stage, astrogliosis can maintain the stabilization of a micro-environment to protect against the neuronal loss and axonal injury. However, persistent astrogliosis will be leading to glial scar formation, the major impediment for axonal regeneration in chronic stage after CNS injury or disease (Saban et al. 2007). Therefore, the ultimate role of PAR-2 for functional recovery depends on the balance between the two ambivalent effects. In our study, PAR-2 exerted its regulating effects on functional recovery possibly by bringing the two ambivalent effects into certain balance, in which inhibition of astrogliosis led to the prevention of glial scar formation. Also certain extent of the rest of astrogliosis had been protected against the neuronal loss and axonal injury. However, the optimum degree of inhibition in astrogliosis and bringing the best functional outcome is unclear and needs further study. The remaining neurons and axonal regeneration, as we know, play a key role in functional recovery after SCI. So, PAR-2 may affect the functional outcomes by its neuroprotective effects and regulating the glial scar formation.

Since, the expression of GFAP and vimentin are directly related to the glial scar formation and JNK signal pathway was involved in the regulating process of the expression of GFAP and vimentin. We detected the p-JNK in our study and found that PAR-2 could regulate its expression from the first day after SCI. Also, the changes in p-JNK expression occurred before the GFAP and vimentin expression changes. Thus, we inferred that PAR-2 exerted its effects via JNK signal pathway. To explore the function of JNK in the expression of GFAP and vimentin, we tried to inhibit these effects of PAR-2 by administering the JNK activator in the presence of inhibition of PAR-2 activity or giving the JNK inhibitor in the presence of promotion of PAR-2 activity. Our results showed that the effects of PAR-2 on the expression of GFAP and vimentin could be reversed by the activator or inhibitor of JNK. At the same time, the functional outcomes evaluated by BBB score had also been reversed. Thus, we concluded that PAR-2 exerts its effects on GFAP and vimentin expression and functional outcomes via JNK signal pathway. These data indicate the possibility of PAR-2 and JNK to be the effective targets to treat SCI.

Many studies reported that PAR-2 had diverse functions related to inflammation in $\mathrm{CNS}$, such as inflammatory cell migration and proinflammatory cytokines production (Ishikawa et al. 2009, Sevigny et al. 2011). Moreover, mounting evidence indicated that inflammation in CNS was involved with the JNK signal pathways (Sofroniew and Vinters 2010). In addition, 
different studies had also reported that inflammatory reaction was involved in reactive astrogliosis and neuroprotective effects (Zhang et al. 2010). Our findings were consistent with the above studies. However, the precise mechanism of PAR-2 on glial scar formation, neurons residues and the regulation of the inflammatory process after SCI is currently unclear and may need further studies.

In conclusion, our study proved for the first time that inhibition of PAR-2 activity reduces the glial scar formation and improves the neurons residues and functional recovery after SCI through inhibiting JNK signaling.

\section{Conflict of Interest}

There is no conflict of interest.

\section{Competing interests}

The authors declare that they have no competing interests.

\section{Acknowledgements}

The authors are grateful for the Neuroscience Research
Center of Chongqing Medical University and all people in that center, especially professor Shan-quan Sun.

\section{Author Contributions}

Conceive and design of the experiments: YY. Perform the experiments: TZL, HD and QL. Analyze the data: QL, YZX. Contribute reagents/materials/analysis tools: TZL, HD and QL. Wrote the paper: TZL, HD, QL and RD.

\section{Ethics approval and consent to participate}

All animal experiments and procedures were approved by the Institutional Animal Care and Use Committees of the First Affiliated Hospital of Chongqing Medical University (CMU-1-2633).

\section{Abbreviations}

SCI - spinal cord injury, PAR-2 - protease-activated receptor-2, CCI - clip compression injury, FS - PAR-2 inhibitor FSLLRY-NH2, SL - PAR-2 activator SLIGRLNH2, AN - anisomycin (a JNK activator), SP - SP600125 (a JNK inhibitor), BBB - Basso, Beattie, and Bresnahan.

\section{References}

BASSO DM: Behavioral testing after spinal cord injury: congruities, complexities, and controversies. J Neurotrauma 21: 395-404, 2004.

BUSHELL T: The emergence of proteinase-activated receptor-2 as a novel target for the treatment of inflammationrelated CNS disorders. J Physiol 581: 7-16, 2007.

GUO RB, WANG GF, ZHAO AP, GU J, SUN XL, HU G: Paeoniflorin pretects against ischemia-induced brain damages in rats via inhibiting MAPKs/NF-кB-mediated inflammatory responses. PLoS One 7: e49701, 2012.

HU R, ZHOU J, LUO C, LIN J, WANG X, LI X, BIAN X, LI Y, WAN Q, YU Y, FENG H: Glial scar and neuroregeneration: histological, functional, and magnetic resonanece imaging analysis in chronic spinal cord injury. J Neurosurg Spine 13: 169-180, 2010.

IDE J, AOKI T, ISHIVATA S, GLUSA E, STRUKOVA SM: Proteinase-activated receptor agonists stimulate the increase in intracellular $\mathrm{Ca}^{2+}$ in cardiomyocytes and proliferation of cardiac fibroblasts from chick embryos. Bull Exp Biol Med 144: 760-763, 2007.

IRVING EA, BARONE FC, REITH AD, HADINGHAM SJ, PARSONS AA: Differential activation of MAPK/ERK and p-38/SAPK in neurons and glial following focal cerebral ischaemia in the rat. Brain Res Mol Brain Res 77: $65-75,2000$.

ISHIKAWA C, TSUDA T, KONISHI H, NAKAGAWA N, YAMANISHI K: Tetracyclines modulate proteaseactivated receptor 2-mediated proinflammatory reactions in epidermal keratinocytes. Antimicrob Agents Chemother 53: 1760-1765, 2009.

LIU X, CHENG C, SHAO B, WU X, JI Y, LIU Y, LU X, SHEN A: CDK11(p58) promotes rat astrocyte inflammatory response via activating p38 and JNK pathways induced by lipopolysaccharide. Neurochem Res 37: 563-573, 2012.

LOHMAN RJ, O'BRIEN TJ, COCKS TM: Protease-activated receptor-2 regulates trypsin expression in the brain and protects against seizures andepileptogenesis. Neurobiol Dis 30: 84-93, 2008. 
LUO W, WANG Y, REISER G: Protease-activated receptors in the brain: receptor expression, activation, and functions in neurodegeneration and neuroprotection. Brain Res Rev 56: 331-345, 2007.

MCCOY KL, TRAYNELIS SF, HEPLER JR: PAR1 and PAR2 couple to overlapping and distinct sets of G proteins and linked signaling pathways to differentially regulate cell physiology. Mol Pharmacol 77: 1005-1015, 2010.

MIZE GJ, WANG W, TAKAYAMA TK: Prostate-specific kallikreins-2 and -4 enhance the proliferation of DU-145 prostate cancer cells through protease-activated receptors-1 and -2. Mol Cancer Res 6: 1043-1051, 2008.

NOORBAKHSH F, TSUTSUI S, VERGNOLLE N, BOVEN LA, SHARIAT N, VODJGANI M, WARREN KG, ANDRADE-GORDON P, HOLLENBERG MD, POWER C: Protease-activated receptor 2 modulates neuroinflammation in experimental autoimmune encephalomyelitis and multiple sclerosis. J Exp Med 203: 425-435, 2006.

OSUGA Y, HIROTA Y, YOSHINO O, HIRATA T, KOGA K, TAKETANI Y: Proteinase-activated receptors in the endometrium and endometriosis. Front Biosci (Schol Ed) 4: 1201-1212, 2012.

PARK GH, RYU JR, SHIN CY, CHOI MS, HAN BH, KIM WK, KIM HC, KO KH: Evidence that protease-activated receptor-2 mediates trypsin-induced reversal of stellation in cultured rat astrocytes. Neurosci Res 54: 15-23, 2006.

PARK GH, JEON SJ, RYU JR, CHOI MS, HAN SH, YANG SI, RYU JH, CHEONG JH, SHIN CY, KO KH: Essential role of mitogen-activated protein kinase pathways in protease activated receptor 2-mediated nitric-oxide production from rat primary astrocytes. Nitric Oxide 21: 110-119, 2009.

PARK GH, JEON SJ, KO HM, RYU JR, LEE JM, KIM HY, HAN SH, KANG YS, PARK SH, SHIN CY, KO KH: Activation of microglial cells via protease-activated receptor 2 mediates neuronal cell death in cultured rat primary neuron. Nitric Oxide 22: 18-29, 2010.

QU WS, TIAN DS, GUO ZB, FANG J, ZHANG Q, YU ZY, XIE MJ, ZHANG HQ, LÜ JG, WANG W: Inhibition of EGFR/MAPK signaling reduces microglial inflammatory response and the associated secondary damage in rats after spinal cord injury. J Neuroinflammation 9: 178, 2012.

RALAY RANAIVO H, WAINWRIGHT MS: Albumin activates astrocytes and microglia through mitogen-activated protein kinase pathways. Brain Res 1313: 222-231, 2010.

SABAN R, D'ANDREA MR, ANDRADE-GORDON P, DERIAN CK, DOZMOROV I, IHNAT MA, HURST RE, SIMPSON C, SABAN MR: Regulatory network of inflammation downstream of proteinase-activated receptors. BMC Physiol 7: 3, 2007.

SEVIGNY LM, ZHANG P, BOHM A, LAZARIDES K, PERIDES G, COVIC L, KULIOPULOS A: Interdicting protease-activated receptor-2-driven inflammation with cell-penetrating pepducins. Proc Natl Acad Sci USA 108: 8491-8496, 2011.

SOFRONIEW MV, VINTERS HV: Astrocytes: biology and pathology. Acta Neuropathol 119: 7-35, 2010.

USUL H, CAKIR E, ARSLAN E, PEKSOYLU B, ALVER A, SAYIN OC, TOPBAS M, BAYKAL S: Effects of clotrimazole on experimental spinal cord injury. Arch Med Res 37: 571-575, 2006.

WANG J, MA C, RONG W, JING H, HU X, LIU X, JIANG L, WEI F, LIU Z: Bog bilberry anthocyanin extract improves motor functional recovery by multifaceted effects in spinal cord injury. Neurochem Res $\mathbf{3 7}$ : 2814-2825, 2012.

XIA Y, ZHAO T, LI J, LI L, HU R, HU S, FENG H, LIN J: Antisense vimentin cDNA combined with chondroitinase $\mathrm{ABC}$ reduces glial scar and systic cavity formation following spinal cord injury in rats. Biochem Biophys Res Commun 377: 562-566, 2008.

ZHANG D, HU X, QIAN L, O'CALLAGHAN JP, HONG JS: Astroglosis in CNS pathologies: is there a role for microglia? Mol Neurobiol 41: 232-241, 2010. 